\title{
Body Weight Result Standard Unit
}

National Cancer Institute

\section{Source}

National Cancer Institute. Body Weight Result Standard Unit. NCI Thesaurus. Code C119793.

The standard unit of measure for body weight results. 